\title{
Perturbation-Theory Machine Learning (PTML) Multilabel Model of the ChEMBL Dataset of Preclinical Assays for Antisarcoma Compounds
}

\author{
Alejandro Cabrera-Andrade, ${ }^{*} \&$ Andrés López-Cortés, ${ }^{\&}$ Cristian R. Munteanu, Alejandro Pazos, \\ Yunierkis Pérez-Castillo, Eduardo Tejera, Sonia Arrasate, and Humbert González-Díaz*
}

Cite This: ACS Omega 2020, 5, 27211-27220

Read Online

ABSTRACT: Sarcomas are a group of malignant neoplasms of connective tissue with a different etiology than carcinomas. The efforts to discover new drugs with antisarcoma activity have generated large datasets of multiple preclinical assays with different experimental conditions. For instance, the ChEMBL database contains outcomes of 37,919 different antisarcoma assays with 34,955 different chemical compounds. Furthermore, the experimental conditions reported in this dataset include 157 types of biological activity parameters, 36 drug targets, 43 cell lines, and 17 assay organisms. Considering this information, we propose combining perturbation theory $(\mathrm{PT})$ principles with machine learning (ML) to develop a PTML model to predict

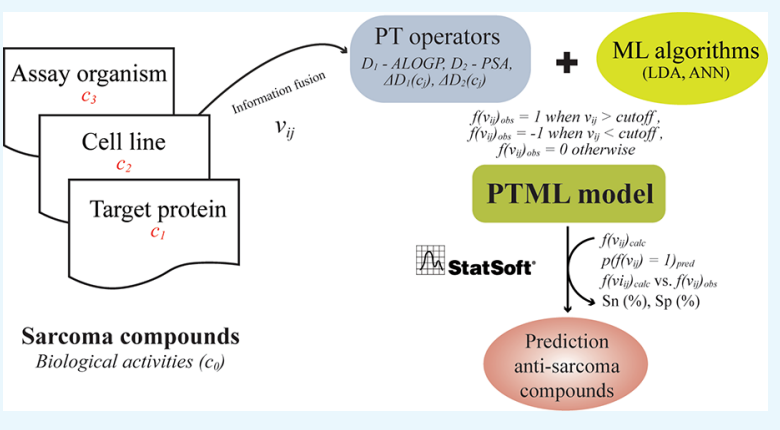
antisarcoma compounds. PTML models use one function of reference that measures the probability of a drug being active under certain conditions (protein, cell line, organism, etc.). In this paper, we used a linear discriminant analysis and neural network to train and compare PT and non-PT models. All the explored models have an accuracy of $89.19-95.25 \%$ for training and $89.22-95.46 \%$ in validation sets. PTML-based strategies have similar accuracy but generate simplest models. Therefore, they may become a versatile tool for predicting antisarcoma compounds.

\section{INTRODUCTION}

Sarcomas are a group of malignant neoplasms of connective tissue. Although their prevalence is much lower than carcinomas, the number of cases is increasing according to the World Health Organization. ${ }^{1}$ At the molecular level, their behavior differs from carcinomas, presenting a more varied and complex etiology. This high etiological complexity possibly stems from their mesenchymal origin, which makes it difficult to propose new therapeutic targets for the respective treatment. $^{2-6}$ Representative anticancer compounds tend to have high cytotoxicity and low cellular specificity. ${ }^{7}$ This leads to a decreased efficiency within the treatment and a low remission rate of the disease. However, a description of new molecular markers and the constant performance of drug preclinical assays have generated large amounts of data. ${ }^{8-12}$ This data, if adequately rationalized, may lead in turn to the design of more selective drugs, which takes into account specific drivers based on pathogenic signaling pathways. For instance, the Chemical Database of the European Molecular Biology Laboratory (ChEMBL) ${ }^{13,14}$ contains experimental outcomes for $>37,900$ different preclinical assays of antisarcoma drug candidates. These assays cover a large and structurally heterogeneous series of $>34,900$ different chemical compounds. Furthermore, the preclinical assays have been carried out on very different experimental conditions. These experimental conditions include up to 155 different types of biological activity parameters, 36 protein targets, 43 cell lines, and 17 assay organisms. Overall, this forms a large and complex dataset susceptible to analysis so as to extract useful knowledge for drug discovery.

In this context, we can use computational techniques to explore this experimental dataset due to the evident difficulties to analyze it manually. Specifically, cheminformatics methodologies have succeeded in the discovery of new drug candidates effective in the wet-lab. ${ }^{15,16}$ However, many models developed thus far are applied only to carcinomas and/or are focused on homologous series of compounds with one target or a single cell line. ${ }^{17-26}$ In recent years, several studies have focused on applying these methodologies to the study of new types of antisarcoma drugs, mainly on cell lines. ${ }^{27-30}$ However, almost all the models reported have a narrow domain of application because they focus on only one set of conditions, for instance,

Received: July 13, 2020

Accepted: October 6, 2020

Published: October 15, 2020 
Table 1. PTML Model Results

\begin{tabular}{|c|c|c|c|c|c|}
\hline \multirow[b]{2}{*}{ series } & \multirow[b]{2}{*}{ statistical parameter $^{a}$} & \multirow[b]{2}{*}{ predicted statistics (\%) } & \multirow[b]{2}{*}{ observed set } & \multicolumn{2}{|c|}{ predicted set } \\
\hline & & & & $f\left(v_{\mathrm{ij}}\right)_{\text {pred }}=0$ & $f\left(v_{\mathrm{ij}}\right)_{\text {pred }}=1$ \\
\hline \multirow[t]{3}{*}{ training } & $\mathrm{Sp}$ & 95.63 & $f\left(v_{\mathrm{ij}}\right)_{\mathrm{obs}}=0$ & 25,647 & 1172 \\
\hline & $\mathrm{Sn}$ & 79.64 & $f\left(v_{\mathrm{ij}}\right)_{\mathrm{obs}}=1$ & 330 & 1291 \\
\hline & Ac & 94.72 & total & 25,977 & 2463 \\
\hline \multirow[t]{3}{*}{ validation } & $\mathrm{Sp}$ & 95.79 & $f\left(v_{\mathrm{ij}}\right)_{\mathrm{obs}}=0$ & 8559 & 376 \\
\hline & $\mathrm{Sn}$ & 81.62 & $f\left(v_{\mathrm{ij}}\right)_{\mathrm{obs}}=1$ & 100 & 444 \\
\hline & Ac & 94.98 & total & 8659 & 820 \\
\hline
\end{tabular}

Table 2. Variables Used to Fit the PTML Model

\begin{tabular}{|c|c|c|c|c|}
\hline $\operatorname{condition}^{a}\left(\mathbf{c}_{\mathbf{j}}\right)$ & $\begin{array}{l}\text { condition } \\
\text { name }\end{array}$ & symbol & operator formula & operator information \\
\hline $\mathrm{c}_{0}$ & activity type & $f\left(v_{\mathrm{ij}}\right)_{\mathrm{obs}}$ & $\begin{array}{l}=\operatorname{IF}\left(\operatorname{AND}\left(v_{\mathrm{ij}}>\operatorname{cutoff}\left(\mathrm{c}_{0}\right), d\left(\mathrm{c}_{0}\right)\right.\right. \\
\quad=1), 1, \operatorname{IF}\left(\operatorname{AND}\left(v_{\mathrm{ij}}<\right.\right. \\
\left.\left.\left.\quad \text { cutoff }\left(\mathrm{c}_{0}\right), d\left(\mathrm{c}_{0}\right)=-1\right), 1,0\right)\right)\end{array}$ & observed classification of the outcome $v_{\mathrm{ij}}$ in the assay with conditions $\mathbf{c}_{\mathrm{j}}$ \\
\hline $\mathrm{c}_{0}$ & activity type & $f\left(v_{\mathrm{ij}}\right)_{\mathrm{ref}}$ & $n\left(f\left(v_{\mathrm{ij}}\right)_{\mathrm{obs}}=1\right) / n_{\mathrm{j}}$ & $\begin{array}{l}\text { function of reference if the observed value of probability } p\left(f\left(\mathrm{v}_{\mathrm{ij}}\right)=1\right)_{\text {expt }} \text { for the activity } v_{\mathrm{ij}} \text { of } \\
\text { type } c_{0}\end{array}$ \\
\hline $\mathbf{c}_{\mathrm{j}}=\left[\mathrm{c}_{1}, \mathrm{c}_{2}, \mathrm{c}_{3}\right]$ & $\begin{array}{l}\text { all } \\
\text { conditions } \\
\left(\mathbf{c}_{\mathrm{j}}\right)\end{array}$ & $\Delta D_{1}\left(\mathbf{c}_{\mathrm{j}}\right)$ & $\operatorname{ALOGP}_{\mathrm{i}}-\left\langle\operatorname{ALOGP}\left(c_{\mathrm{j}}\right)\right\rangle$ & $\begin{array}{l}\text { deviation of the molecular descriptors of hydrophobicity/lipophilicity } D_{1}(\text { ALOGP }) \text { and } \\
\text { polar surface area } D_{2} \text { (PSA) from each expected value }\left(\left\langle D_{1}\left(c_{\mathrm{j}}\right)\right\rangle\right) \text { or }\left(\left\langle D_{2}\left(\mathbf{c}_{\mathrm{j}}\right)\right\rangle\right) \text { for the } \\
\text { conditions } \mathbf{c}_{\mathrm{j}}\left(\mathrm{c}_{1}=\text { protein target; } c_{2}=\text { cell line; } c_{3}=\text { assay organism }\right)\end{array}$ \\
\hline $\mathrm{c}_{\mathrm{j}}=\left[\mathrm{c}_{1}, \mathrm{c}_{2}, \mathrm{c}_{3}\right]$ & $\begin{array}{l}\text { all } \\
\text { conditions } \\
\left(\mathbf{c}_{\mathrm{j}}\right)\end{array}$ & $\Delta D_{2}\left(\mathbf{c}_{\mathfrak{j}}\right)$ & $\mathrm{PSA}_{\mathrm{i}}-\left\langle\operatorname{PSA}\left(\mathrm{c}_{\mathrm{j}}\right)\right\rangle$ & \\
\hline
\end{tabular}

one specific property, target protein, or cell line. Thus, models where multiple conditions of assays are considered at the same time are attractive. Perturbation theory (PT) ideas with machine learning $(\mathrm{ML})$ methods (PT $+\mathrm{ML}=\mathrm{PTML}$ models) are particularly useful for fitting complex datasets with big data features in drug discovery, proteomics, nanotechnology, etc. $^{31-41}$

PTML models begin with one function of reference that measures the probability of a drug to be active under certain conditions (protein, cell line, organism, etc.). Next, PTML models use PT operators (PTOs) to account for the perturbations (deviations) of the input variables of this drug with respect to a population of drugs assayed under the same conditions. ML algorithms are used to establish the relationship between the inputs and the output variable. In cancer research, Speck-Planche et al. and other researchers have developed PTML-like models for different types of cancers (with an emphasis on carcinomas) such as bladder, prostate, brain, and breast cancers. ${ }^{42-50}$ In addition, Bediaga et al. developed a PTML algorithm for predicting anticancer compounds using data for multiple types of carcinomas at the same time. ${ }^{51}$ Speck-Planche et al. also recently developed the first PTML-like model for the prediction of antisarcoma compounds using a spectral moment approach. ${ }^{52}$

In any case, there are no reports of other PTML-like models for antisarcoma compounds. In this study, we carried out a comprehensive compilation, curation, and preprocessing of the ChEMBL dataset for preclinical assays of antisarcoma compounds. After that, we developed the first PTML model able to fit this complex dataset with $>37,900$ assays and $>34,900$ compounds. To the best of our knowledge, the study outperforms all previous efforts in terms of simplicity of the model and number of cases, compounds, and cell lines considered.

\section{RESULTS AND DISCUSSION}

PTML Antisarcoma Compound Model. The statistical parameters for the PTML model showed a high specificity $(\mathrm{Sp})$ and sensitivity $(\mathrm{Sn})$ for the training series (95.63 and 79.64 , respectively). In addition, similar values were obtained for $S p$ (95.79) and $S n$ (81.62) in the validation sets. Furthermore, the p-level obtained from the chi-square $\left(\chi^{2}=\right.$ 16848.08) was $<0.05$, indicating that the model is able to perform a statistically significant separation of both classes. It is also interesting to observe the high overall accuracy (Ac) obtained in both sets: over 94\% (Table 1). These results suggest that the generated model performs a statistically significant classification of antisarcoma compounds; hence, it can be considered useful for classification models with application in medicinal chemistry. The full list of biological activities $\left(c_{0}\right)$ in the ChEMBL dataset of antisarcoma preclinical experimental assays is shown in Table S1.

The resulting PTML-linear discriminant analysis (LDA) model showed the following formula

$$
\begin{aligned}
f\left(v_{\mathrm{ij}}\right)_{\mathrm{calc}}= & -11.8545+34.8028 \cdot f\left(v_{\mathrm{ij}}\right)_{\mathrm{ref}}+0.37 \cdot D_{1} \\
& -0.0128 \cdot D_{2}-0.3616 \cdot\left[D_{1}-\left\langle D_{1}\left(\mathbf{c}_{\mathrm{j}}\right)\right\rangle\right] \\
& +0.0191 \cdot\left[D_{2}-\left\langle D_{2}\left(\mathbf{c}_{\mathrm{j}}\right)\right\rangle\right] \\
n= & 34955, \chi^{2}=16848.08, p<0.001
\end{aligned}
$$

The PTML-LDA model was initiated by using as an input the values the function of reference $f\left(v_{\mathrm{ij}}\right)_{\text {ref }}$ for each compound and by adding the effect of perturbations within the system. These perturbation effects refer to the PTOs $\Delta D_{k}\left(\mathbf{c}_{\mathfrak{j}}\right)$. In eq 1 , " $i$ " and " $j$ " are the assay and condition, respectively. Additional coefficients and terms are described in Table 2.

The parameters ALOGP and PSA are widely used in medicinal chemistry because they are related to the lipophilicity of drugs and, consequently, to their capacity to pass through biological membranes or interact with protein 
Table 3. Comparison to Other PTML Models of Anticancer Compounds

\begin{tabular}{|c|c|c|c|c|c|c|c|}
\hline cancer type $^{a}$ & $\mathrm{PT}^{b}$ & $\mathrm{ML}^{c}$ & $\mathrm{NV}^{d}$ & $\operatorname{cases}^{e}$ & $\operatorname{Sn}(\%)^{f}$ & $\mathrm{Sp}(\%)^{f}$ & ref \\
\hline \multicolumn{8}{|l|}{ sarcoma } \\
\hline MSS & MMA & LDA & 3 & 37,919 & $\sim 80$ & $>90$ & this work \\
\hline MSS & MA & LDA & $>10$ & 3017 & $>90$ & $>90$ & 52 \\
\hline \multicolumn{8}{|l|}{ carcinoma } \\
\hline bladder & MA & LDA & $>10$ & 664 & $>90$ & $>90$ & 44 \\
\hline bladder & & ANN (RBF) & 10 & 664 & $>95$ & $>95$ & 44 \\
\hline breast & MA & LDA & $>10$ & 2272 & $>85$ & $>90$ & 47 \\
\hline colorectal & MA & LDA & $>10$ & 1651 & $>90$ & $>90$ & 46 \\
\hline colorectal & MA & ANN (RBF) & $>10$ & 1651 & $>90$ & $>90$ & 46 \\
\hline prostate & MA & LDA & $>10$ & 1668 & $>85$ & $>90$ & 49 \\
\hline MCS & MMA & LDA & $>10$ & 116,934 & $>70$ & $\sim 90$ & 51 \\
\hline MCS & MMA & LDA & 3 & 116,934 & $>70$ & $>90$ & 51 \\
\hline MCS & MMA & ANN & 4 & 116,934 & $>80$ & $>80$ & 51 \\
\hline
\end{tabular}

${ }^{a}$ MSS, multiple sarcoma subtypes; MCS, multiple carcinoma subtypes. ${ }^{b} \mathrm{PT}$ operators used in PTML models: MMA, multicondition moving average; MA, moving average. ${ }^{c} \mathrm{ML}$ method used for the PTML models: LDA, linear discriminant analysis; ANN, artificial neural networks; RBF, radial basis function; LNN, linear neural networks; E-ANN (RBF), ensemble of artificial neural networks based on the RBF architecture. ${ }^{d} \mathrm{NV}$, number of input variables. ${ }^{e}$ Number of preclinical assays. ${ }^{f}$ Approximate values for training series.

Table 4. Different Scores Calculated for the Selected Biological Activities $\left(c_{0}\right)$

\begin{tabular}{|c|c|c|c|c|c|c|}
\hline activity parameter for $v_{\mathrm{ij}}\left(\mathrm{c}_{0}\right)$ (unit) & $n_{\mathrm{j}}\left(\mathrm{c}_{0}\right)^{a}$ & $\left\langle v_{\mathrm{ij}}\left(\mathrm{c}_{0}\right)\right\rangle^{b}$ & $d_{j}\left(c_{0}\right)^{c}$ & cutoff $\left(c_{0}\right)$ & $n\left(f\left(v_{\mathrm{ij}}\right)_{\mathrm{obs}}=1\right)^{d}$ & $p\left(f\left(v_{\mathrm{ij}}\right)_{\mathrm{obs}}=1 / \mathrm{c}_{0}\right)^{e}$ \\
\hline potency $(\mathrm{nM})$ & 31,581 & 19669.199 & -1 & 100 & 149 & 0.005 \\
\hline $\mathrm{IC}_{50}(\mathrm{nM})$ & 1808 & 228362.82 & -1 & 100 & 177 & 0.098 \\
\hline inhibition (\%) & 690 & 39.186507 & 1 & 50 & 225 & 0.326 \\
\hline $\mathrm{CC}_{50}(\mathrm{nM})$ & 450 & 134445.04 & -1 & 100 & 4 & 0.009 \\
\hline activity (\%) & 404 & 52.416163 & 1 & 50 & 208 & 0.515 \\
\hline $\mathrm{EC}_{50}(\mathrm{nM})$ & 379 & 63578.521 & -1 & 100 & 44 & 0.116 \\
\hline $\mathrm{T} / \mathrm{C}$ & 173 & 26.556832 & 1 & 50 & 28 & 0.162 \\
\hline $\mathrm{IC}_{50}\left(\mu \mathrm{g} \mathrm{mL}^{-1}\right)$ & 167 & 64.429402 & -1 & 60 & 118 & 0.707 \\
\hline $\mathrm{T} / \mathrm{C}(\%)$ & 144 & 156.92153 & 1 & 50 & 123 & 0.854 \\
\hline $\mathrm{GI}_{50}(\mathrm{nM})$ & 113 & 66515.131 & -1 & 100 & 13 & 0.115 \\
\hline $\mathrm{EC}_{50}\left(\mu \mathrm{g} \mathrm{mL}^{-1}\right)$ & 90 & 60.733562 & -1 & 60 & 57 & 0.633 \\
\hline
\end{tabular}

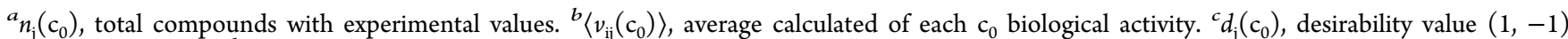
assigned to each $c_{0} \cdot{ }^{d} n\left(f\left(v_{\mathrm{ij}}\right)_{\mathrm{obs}}=1\right)$, total number of biologically active compounds observed within each $\mathrm{c}_{0}$ according to the experimental values $v_{\mathrm{ij}}\left(\mathrm{c}_{0}\right)$ reported for the parameters $\mathrm{j} .{ }^{e} p\left(f\left(v_{\mathrm{ij}}\right)_{\mathrm{obs}}=1 / \mathrm{c}_{0}\right)$, probability of a desired biological activity within the conditions $\mathrm{c}_{0}$.

hydrophobic pockets. ${ }^{53-56}$ The PTML algorithm has been previously applied to the study of multiple preclinical assays of anticancer drugs. As shown in Table 3, most applications have been directed toward the most prevalent carcinomas among the global population. For instance, Speck-Planche et al. reported PTML-like models for bladder, ${ }^{44}$ colorectal, ${ }^{46}$ breast, $^{47}$ prostate $^{49}$ cancers and for multiple carcinoma subtypes. ${ }^{51}$ In addition, PTML-like models have been tested in antibrain tumor agents. ${ }^{45}$ Interestingly, Bediaga et al. demonstrated the application of a PTML on several types of carcinomas simultaneously and obtained similar Sn and Sp values as we did $(>90 \%) .{ }^{51}$ All these PTML-like models are able to account for changes in target proteins, cellular lines, organisms, etc. However, they are specific models for carcinomas, not for sarcomas.

It is worth noting that to the best of our knowledge, SpeckPlanche et $a .^{52}$ seem to be the only researchers to have reported a previous PTML-like model for sarcomas thus far. In their study, the prediction model in external validation resulted in $A c$ (90.78) and $S p$ (90.65) values that were lower than what was obtained in our model $(\mathrm{Ac}=94.98$ and $\mathrm{Sp}=95.79)$. However, our PTML algorithm showed a lower sensitivity in external validation data $(81.62 \%)$ than the model obtained by
Speck-Planche et al. (91.74\%). Even when our model had a much lower number of variables and used a stricter cut-off definition for activity class (i.e., $\mathrm{IC}_{50}=0.1 \mu \mathrm{M}$ instead $1 \mu \mathrm{M}$ ), these aspects alone cannot explain the sensitivity reduction.

The generated PTML-LDA model (eq 1) has important characteristics that allow it to be used within research focused on drug discovery. One of the main advantages of our model is the considerable reduction of input variables for the construction of the algorithm through the inclusion of PTOs. This reduction allowed us to work on datasets with a large amount of information, to define cut-off values, and to calculate the probability of belonging to a class, whether this was a prediction for active compounds (1) or inactive compounds (0). In this way, the $\mathrm{Sn}$ or $\mathrm{Sp}$ values of the model can be adjusted according to the delimited cut-offs. An ideal prediction model has a reasonable trade-off between $\mathrm{Sn}$ and Sp. This means that a high sensitivity is achieved by accepting a relatively low $\mathrm{Sp}$ and, conversely, a high $\mathrm{Sp}$ is reached by compromising $\mathrm{Sn}$. Sp is synonymous with a truenegative rate, which is related to the false-positive rate, ${ }^{30}$ so a high specificity in a prediction model for drug discovery implies that it is unlikely to get a positive result in a drug that does not have a desired biological activity. Thus, a positive 


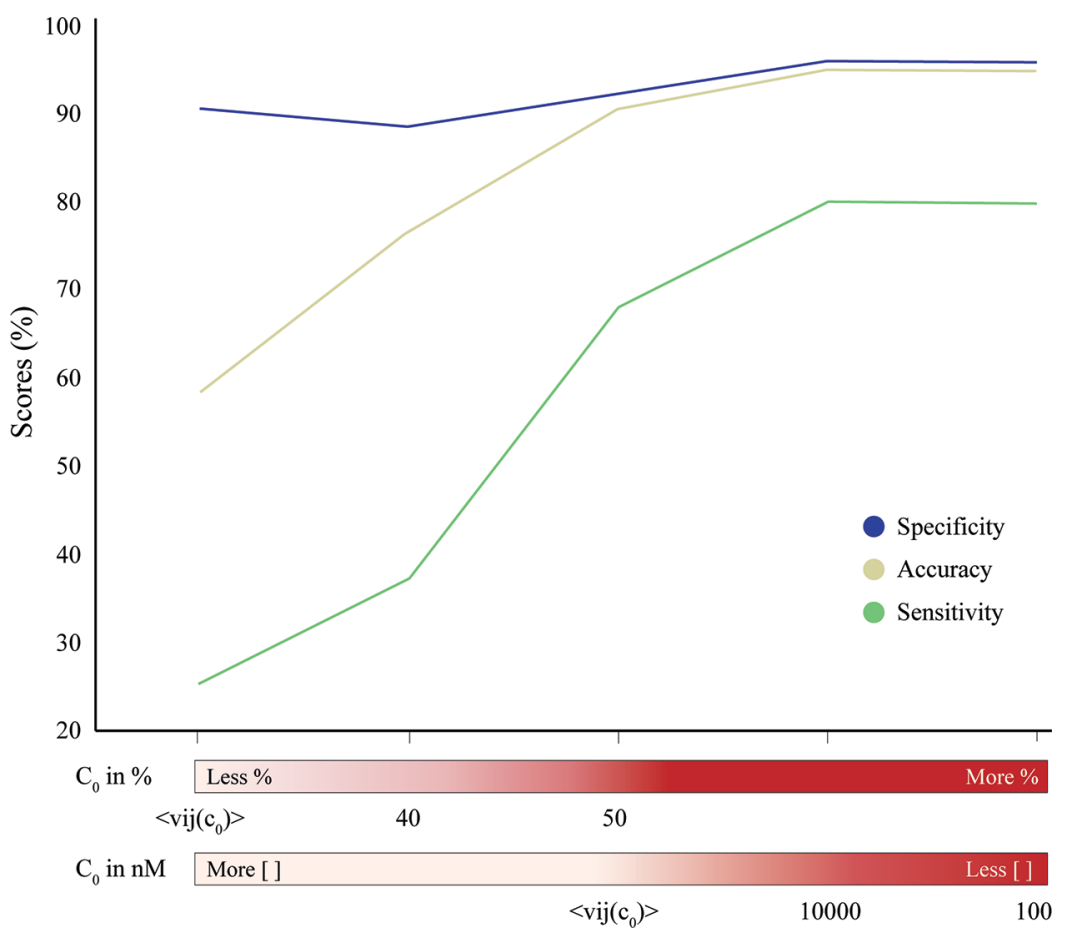

Figure 1. Variation of the specificity, sensitivity, and accuracy values according to the cut-offs implemented. The variation of these scores based on the biological activities $c_{0}$ is included in the $x$-axis. Biological activities $c_{0}$ expressed in \% (e.g., inhibition, activity, tumor growth inhibition, etc.) and those expressed in $\mathrm{nM}$ (e.g., potency, $\mathrm{IC}_{50}, \mathrm{CI}_{50}$, etc.) are described. The final model is obtained by applying cut-off values of 50 for $\mathrm{c}_{0}$ expressed in $\%$ and 100 for $\mathrm{c}_{0}$ expressed in $\mathrm{nM}$.

outcome in a specific model is quite informative in a drug discovery scenario.

On the other hand, a main attribute is the possible combination of several experimental conditions for the prediction of new compounds. In this sense, Speck-Planche et al. ${ }^{52}$ used around 3000 interactions derived from 14 cell lines and only considered $\mathrm{IC}_{50}$ assays for their model. However, we modeled 37,919 interactions cases comprising 36 protein targets, 43 cell lines, and 17 assay organisms. We also included several different assay types (Table 4). The modeling task we have is more complex not only because of the increment in the chemical diversity but also the wide type of heterogeneity in the interactions (i.e., target types and organisms). The two models cannot be compared in this scenario and our reduction in the ability to detect the true-positive cases $(\mathrm{Sn})$ could be a consequence of this data complexity and also the modeling strategy.

PTML Cut-Off Scanning Study. As mentioned above, the cut-off implemented in the model is a rigorous value that, at the experimental level, is important if one desires to increase effectiveness in the process of discovering antisarcoma drugs. A restricted value promotes high certainty in the prediction of active compounds for achieving a desired biological action under multiple test conditions. ${ }^{57-59}$ Furthermore, a strict cutoff can decrease the rate of predicted false positives; therefore, if the assay is to be implemented, then it needs a higher sensitivity or higher specificity. This value can be modeled depending on the experimental conditions one wishes to apply. This cut-off value also influences the accuracy within our model. As observed in Figure 1, when using the average $\left\langle v_{\mathrm{ij}}\left(\mathrm{c}_{0}\right)\right\rangle$ calculated for each $\mathrm{c}_{0}$, the Ac is not a desirable score. These low statistical values are mainly influenced by the low $\mathrm{Sn}$ in the prediction. By increasing the rigor, the model improves its prediction values for the active compounds (1). When looking at these results, our prediction algorithm not only takes into account several experimental conditions but also restricts the prediction of compounds to those that have true biological activity.

PTML vs ML Model Comparison. Most multitasking or multilabel ML methods are useful for predicting multiple categorical outputs for the same set of input continuous variables. ${ }^{60,61}$ However, our problem was a little different: we had to develop an ML model with only two possible outputs, $f\left(v_{\mathrm{ij}}\right)_{\text {pred }}=1$ or 0 , for the same set of input variables. That meant that our model was not multitasking for a single case with a set of input variables containing multiple continuous variables plus multiple categorical input variables. However, we had multiple combinations of input categorical variables or levels for the same set of input continuous variables. Hence, our model was multilabel in the input categorical variables for the same set of input continuous variables. To illustrate this fact, we developed here a comparison of our PTML-LDA model vs classic $\mathrm{ML}$ using multiple labeling categorical variables. As seen in Figure 2A, the performance of our PTML-LDA model compared to a classic ML-LDA demonstrates similar values based on $\mathrm{Sp}, \mathrm{Sn}$, and Ac. Similarly, when developing neural networks (NN), the results of PTML-NN (Figure 2B) and ML-NN (Figure 2C) are quite similar. One of the advantages of our PTML model is the inclusion of PTOs, which greatly reduces the number of variables to generate the algorithm. Thus, although the statistics of all the models generated are quite similar, the PTML methodology allows for the reduction of variables from 164 variables in classic ML methods to only 5 in the PTML model. All the PTML and non-PTML model results are described in Table S2. 
A)

Imput Variables

\begin{tabular}{|c|c|}
\hline$\overleftrightarrow{a}$ & $\begin{array}{l}\mathrm{f}\left(\mathrm{v}_{\left.\mathrm{v}_{\mathrm{i}}\right)_{\mathrm{ef}}}\left(\mathrm{D}_{1}\left(\mathrm{c}_{\mathrm{j}}\right), \mathrm{D}_{2}\left(\mathrm{c}_{\mathrm{j}}\right), \Delta \mathrm{D}_{1}\left(\mathrm{c}_{\mathrm{j}}\right), \Delta \mathrm{D}_{2}\left(\mathrm{c}_{\mathrm{j}}\right)\right.\right.\end{array}$ \\
\hline E & $\begin{array}{l}\mathrm{f}\left(\mathrm{v}_{\mathrm{i}}\right)_{\text {nef }} \\
\mathrm{D}_{1}\left(\mathrm{c}_{\mathrm{j}}\right), \mathrm{D}_{2}\left(\mathrm{c}_{\mathrm{j}}\right), \Delta \mathrm{D}_{1}\left(\mathrm{c}_{\mathrm{j}}\right), \Delta \mathrm{D}_{2}\left(\mathrm{c}_{\mathrm{j}}\right) \\
\mathrm{D}_{3} \cdots{ }_{15}\left(\mathrm{c}_{\mathrm{j}}\right)\end{array}$ \\
\hline
\end{tabular}
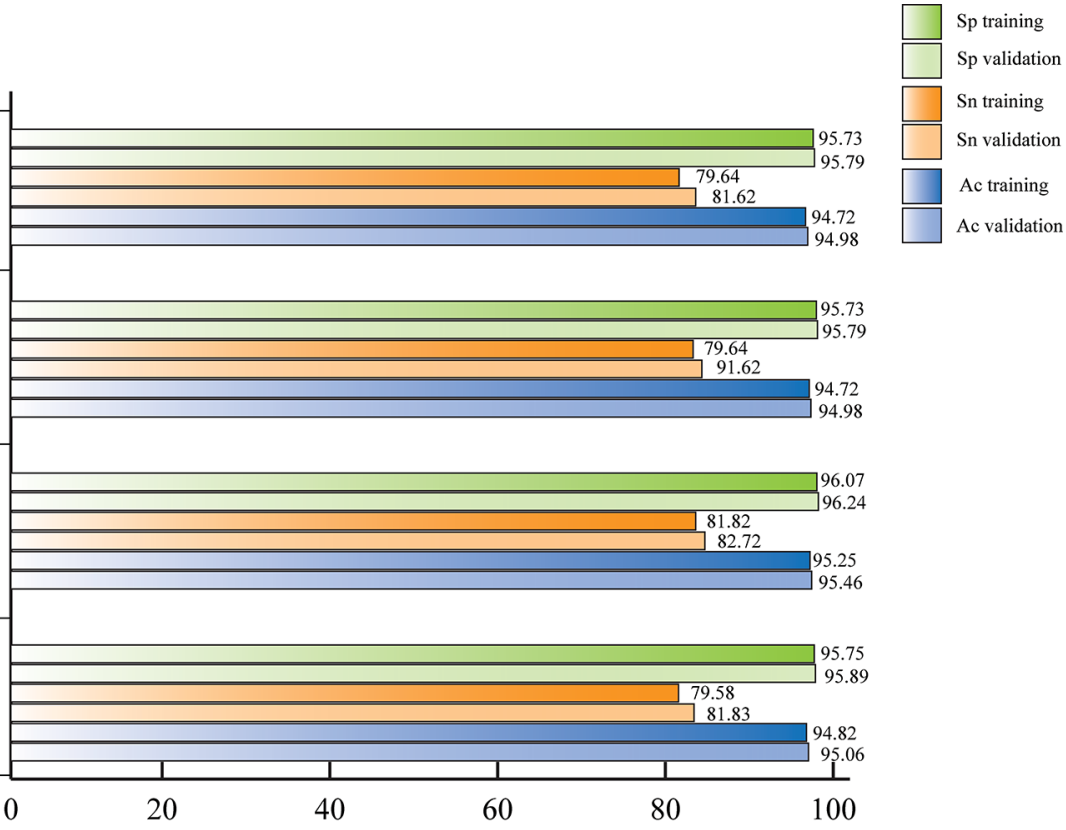

B)

\begin{tabular}{|c|c|}
\hline 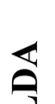 & $\begin{array}{l}D_{1}\left(c_{j}\right), D_{2}\left(c_{j}\right) \\
c_{0}=157(97), c_{1}=20(16), \\
c_{2}=43(32), c_{3}=17(15)\end{array}$ \\
\hline$\Sigma$ & $\begin{array}{l}\mathrm{D}_{1}\left(\mathrm{c}_{\mathrm{j}}\right), \mathrm{D}_{2}\left(\mathrm{c}_{\mathrm{j}}\right) \\
\mathrm{c}_{0}=157(97), \mathrm{c}_{1}=20(16), \\
\mathrm{c}_{2}=43(32), \mathrm{c}_{3}=17(15) \\
\mathrm{D}_{3} \cdots{ }_{15}\left(\mathrm{c}_{\mathrm{j}}\right)\end{array}$ \\
\hline
\end{tabular}

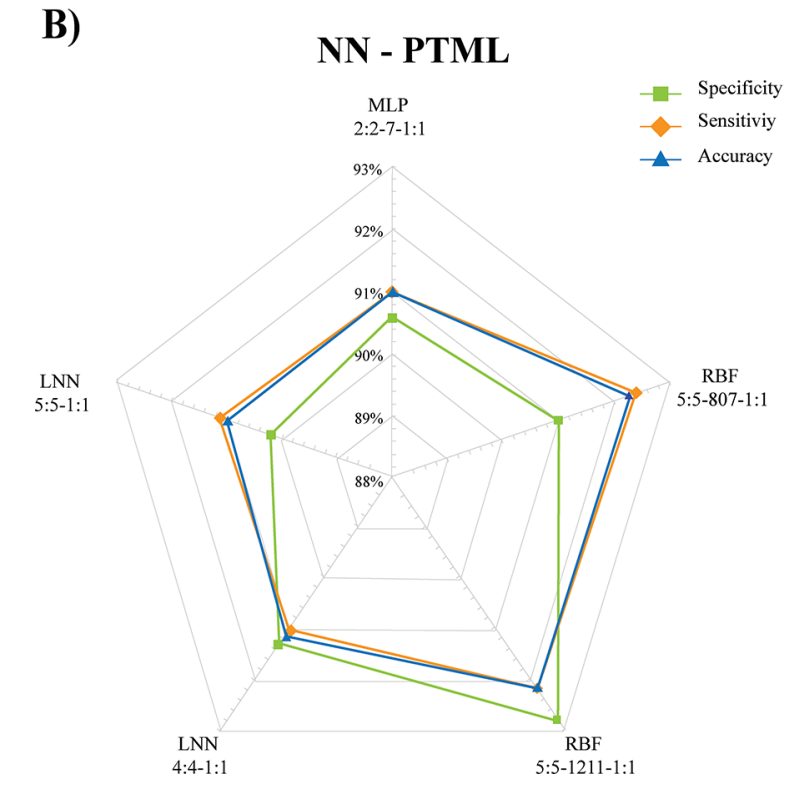

C)
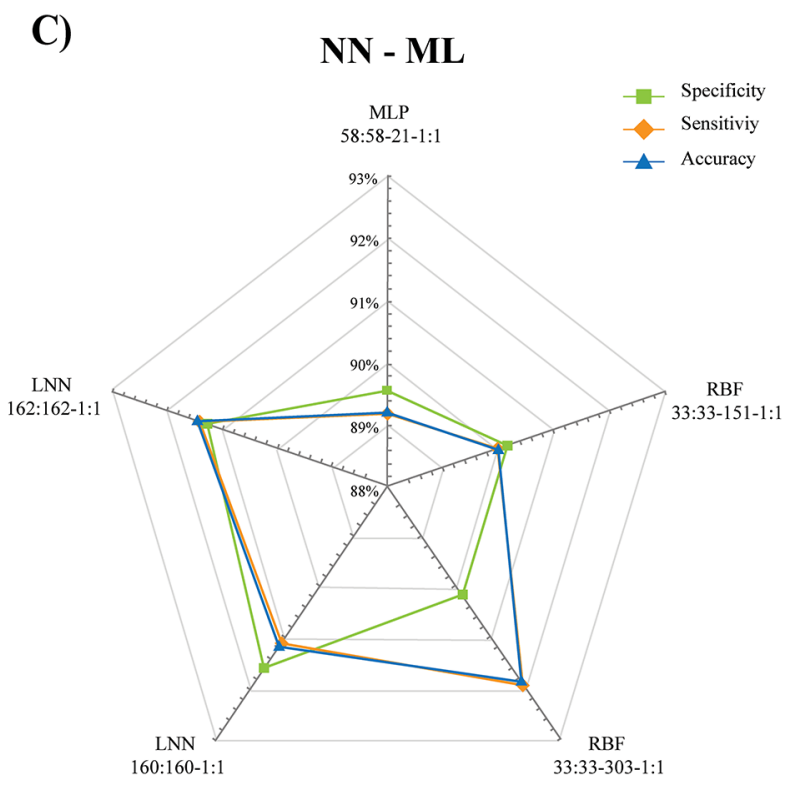

Figure 2. PTML vs ML models. Comparison of sensitivity, specificity, and accuracy of all the generated models. (A) Prediction values of PTMLLDA and ML-LDA models using different types of input variables: $f\left(v_{\mathrm{ij}}\right)_{\text {pred }}$ is the function of reference; $D_{1}\left(\mathbf{c}_{\mathrm{j}}\right)$ and $D_{2}\left(\mathbf{c}_{\mathbf{j}}\right)$ are the ALOGP and PSA descriptors, respectively; $\Delta D_{1}\left(\mathbf{c}_{\mathbf{j}}\right)$ and $\Delta D_{1}\left(\mathbf{c}_{\mathbf{j}}\right)$ are the deviations of the molecular descriptors of ALOGP and PSA, respectively; $D_{3}, \ldots, D_{15}\left(\mathbf{c}_{\mathbf{j}}\right)$ are the 12 BCUT molecular descriptors calculated from ChemAxon. Unlike the PTML model, the ML model is calculated with conditions $c_{1}, c_{2}$, and $c_{3}$ as a separated set of categorical variables. (B) Prediction values between the neural network-PTML (NN-PTML) and (C) NN-ML models. The NN obtained were multilayer perceptron (MLP), linear neural network (LNN), and radial basis function network (RBF).

PTML vs ML Model with Other Descriptors. Previous studies have considered a wide variety and quantity of molecular descriptors in PTML models. For example, for sarcoma modeling, Speck-Planche et al. ${ }^{52}$ used 423 descriptors followed by a feature selection strategy. Similarly, 289 descriptors were used in a PTML model on breast cancer. ${ }^{47}$ We used this approach as a strategy to compare the performance of PTML model vs classic ML techniques including new molecular descriptors (Figure 2A). In this ML study, we included 12 BCUT molecular descriptors $\left(D_{k}\right.$, with $k$ $>2$ ) as an input, which were not used in the previous model, and 162 categorical (dummy) variables $\left(C_{k}\right)$. These $C_{k}$ have been used to label the multiple conditions of the assays $\mathbf{c}_{j}$ (organisms, proteins, cell lines, etc.). One must remember that $D_{1}=$ ALOGP and $D_{2}=$ PSA. The new molecular descriptors were $D_{3}, D_{4}, \ldots, D_{14}$. The expansion of the variables together with the ML strategies yielded good results but did not outperform what was obtained for the PTML-LDA antisarcoma model (as seen in Figure 2A and Table S2) and the number of variables increased to 174 input variables in total. This suggests that by adding different molecular descriptors and probably feature selection strategies, acceptable models for drug discovery can be built. However, our PTML-LDA model based on $D_{1}$ and $D_{2}$ is a simpler yet effective model. 
Table 5. Multiple-Condition Averages for All Sarcoma Assays

\begin{tabular}{|c|c|c|c|c|c|}
\hline \multicolumn{3}{|c|}{ assay condition $\left(\mathbf{c}_{\mathbf{j}}\right)^{a}$} & \multicolumn{3}{|c|}{ parameter } \\
\hline $\mathrm{c}_{1}=$ protein $($ gene $)$ & $c_{2}=$ cell line & $c_{3}=$ assay organisms ${ }^{b}$ & $n_{\mathrm{j}}\left(\mathbf{c}_{\mathrm{j}}\right)$ & $\left\langle D_{1}\left(c_{j}\right)\right\rangle$ & $\left\langle D_{2}\left(\mathbf{c}_{\mathrm{j}}\right)\right\rangle$ \\
\hline O75874 (IDH1) & $\mathrm{MD}$ & H. sapiens & 31,581 & 3.778 & 70.597 \\
\hline $\mathrm{MD}$ & $\mathrm{MD}$ & M. musculus & 1440 & 2.67 & 103.712 \\
\hline $\mathrm{MD}$ & $\mathrm{U} 2 \mathrm{OS}$ & H. sapiens & 746 & 4.421 & 78.325 \\
\hline $\mathrm{MD}$ & HOS & H. sapiens & 637 & 3.603 & 89.517 \\
\hline MD & $\mathrm{MD}$ & H. sapiens & 375 & 3.846 & 69.876 \\
\hline MD & SAOS-2 & H. sapiens & 358 & 4.882 & 81.659 \\
\hline $\mathrm{MD}$ & Sarcoma-180 & M. musculus & 271 & 1.108 & 83.68 \\
\hline $\mathrm{MD}$ & MG-63 & H. sapiens & 241 & 2.965 & 111.864 \\
\hline $\mathrm{MD}$ & M5076 & M. musculus & 197 & 3.033 & 114.886 \\
\hline MD & НТ-1080 & H. sapiens & 170 & 2.826 & 97.731 \\
\hline MD & 143B & H. sapiens & 131 & 1.283 & 141.735 \\
\hline $\mathrm{MD}$ & $\mathrm{MD}$ & Pseudomonas aeruginosa & 130 & 0.277 & 142.432 \\
\hline MD & $\mathrm{MD}$ & $\mathrm{MD}$ & 126 & 1.898 & 93.448 \\
\hline $\mathrm{MD}$ & rhabdomyosarcoma cell & H. sapiens & 116 & 4.036 & 77.177 \\
\hline $\mathrm{MD}$ & CCRF S $^{-180}$ & M. musculus & 109 & 0.978 & 140.984 \\
\hline $\mathrm{P} 13053(V d r)$ & $\mathrm{MD}$ & Rattus norvegicus & 64 & 5.844 & 60.476 \\
\hline $\mathrm{MD}$ & MES-SA & H. sapiens & 64 & 2.956 & 89.631 \\
\hline $\mathrm{MD}$ & $\mathrm{MD}$ & RSV & 61 & 1.277 & 127.944 \\
\hline $\mathrm{MD}$ & 6C3HED & M. musculus & 60 & 3.09 & 97.831 \\
\hline MD & $\mathrm{C} 3 \mathrm{H} / 3 \mathrm{~T} 3$ & MMSV & 50 & 0.327 & 139.359 \\
\hline P35354 (PTGS2) & $\mathrm{MD}$ & H. sapiens & 49 & 3.515 & 69.152 \\
\hline $\mathrm{MD}$ & A204 & H. sapiens & 44 & 1.189 & 106.655 \\
\hline P03359 (pol) & $\mathrm{MD}$ & WMSV & 44 & 6.786 & 204.629 \\
\hline $\mathrm{MD}$ & $\mathrm{MD}$ & Gallus gallus & 43 & 0.516 & 106.529 \\
\hline P37231 (PPARG) & MD & H. sapiens & 40 & 5.33 & 83.835 \\
\hline $\mathrm{MD}$ & $\mathrm{MD}$ & MMSV & 39 & 0.213 & 166.782 \\
\hline Q07869 (PPARA) & $\mathrm{MD}$ & H. sapiens & 37 & 5.364 & 81.891 \\
\hline Q13443 (ADAM9) & $\mathrm{MD}$ & H. sapiens & 35 & 2.914 & 91.186 \\
\hline MD & MD & R. norvegicus & 34 & 5.245 & 64.58 \\
\hline $\mathrm{MD}$ & fibroblast & MMSV & 33 & -1.224 & 150.956 \\
\hline $\mathrm{MD}$ & $\mathrm{MD}$ & enterovirus & 33 & 6.348 & 38.332 \\
\hline $\mathrm{MD}$ & $\mathrm{MD}$ & human herpesvirus 1 & 31 & 6.27 & 57.306 \\
\hline $\mathrm{MD}$ & $791 \mathrm{~T}$ cell line & H. sapiens & 28 & -1.179 & 139.194 \\
\hline $\mathrm{MD}$ & $\mathrm{C} 3 \mathrm{H} / 3 \mathrm{~T} 3$ & M. musculus & 28 & 1.745 & 115.047 \\
\hline P08253 (MMP2) & $\mathrm{MD}$ & H. sapiens & 28 & 3.31 & 112.85 \\
\hline $\mathrm{MD}$ & $\mathrm{MD}$ & human enterovirus 71 & 28 & 1.967 & 124.221 \\
\hline P04637 (TP53), Q00987 (MDM2) & SJSA-1 & H. sapiens & 27 & 5.213 & 49.453 \\
\hline P06401 (PGR) & $\mathrm{MD}$ & H. sapiens & 26 & 4.494 & 32.958 \\
\hline $\mathrm{MD}$ & HL-60 & H. sapiens & 25 & 3.81 & 33.754 \\
\hline
\end{tabular}

${ }^{a} \mathrm{MD}$, missing data. ${ }^{b} \mathrm{RSV}$, Rous sarcoma virus; MLV, murine leukemia virus; MMSV, Moloney murine sarcoma virus; WMSV, Woolly monkey sarcoma virus.

Multiple-Condition Averages in the PTML Antisarcoma Model. In total, we found 83 possible combinations of multiple conditions for all the included sarcoma assays. As shown in Table 5 , the $n_{j}\left(c_{j}\right)$ with the highest number of entries corresponded to tests on human cell lines and on cell lines in Mus musculus. The multicondition moving averages (MMAs) used here, $\left\langle D_{1}\left(\mathbf{c}_{\mathfrak{j}}\right)\right\rangle$ and $\left\langle D_{2}\left(\mathbf{c}_{\mathfrak{j}}\right)\right\rangle$, vary significantly along all combinations. However, the anticancer compounds observed for the human osteosarcoma cell lines U2OS, HOS, SAOS-2, MG-63, and 143B and for the fibrosarcoma cell line HT-1080 were in a range of $\left\langle D_{1}\left(\mathbf{c}_{\mathfrak{j}}\right)\right\rangle$ of 1.2-3.7. A similar range was observed in compounds tested in M. musculus $\left(\left\langle D_{1}\left(\mathbf{c}_{\mathrm{j}}\right)\right\rangle=1-\right.$ 3). Interestingly, when comparing these values with the variation of $\left\langle D_{2}\left(\mathbf{c}_{\mathfrak{j}}\right)\right\rangle$, tests on virus lines, such as Moloney murine sarcoma virus and Woolly monkey sarcoma virus, had higher means (between 140 and 205). Since the ALOGP coefficient is a measure widely used in drug discovery to assess the degree of absorption, distribution in the body, penetration across biological membranes, metabolism, and excretion, this range identified in our results is an important space for the prediction of antisarcoma drugs. ${ }^{62,63}$ Likewise, the range of PSA evidenced in viral line assays may be a better space for this coefficient if it is desired to predict new compounds in these experimental conditions. This may be interesting when defining the validation of a certain antisarcoma compound. Thus, if a compound is significantly predicted in an experimental animal or human cell lines, then it will be possible to propose validations at the preclinical level or in clinical trials, respectively.

How to Use the PTML Model in Practice. The model is capable of scoring the activity of a single compound under different assay conditions. To predict a new compound, first, we have to substitute the expected values of function of reference $f\left(v_{\mathrm{ij}}\right)_{\mathrm{ref}}=p\left(f\left(v_{\mathrm{ij}}=1\right)_{\text {expt }}\right.$ in the model. As 
aforementioned, this is the probability of the compound being active for a given biological activity parameter $\left(c_{0}\right)$ (see Table 2). Next, we need to substitute into the equation the values of molecular descriptors $D_{1}=$ ALOGP and $D_{2}=$ PSA of the compound (chemical structure), calculated with the same algorithm used in the ChEMBL dataset. Last, we have to substitute into the equation the average values (expected values) of the molecular descriptors $\left\langle D_{1}\left(c_{j}\right)\right\rangle$ for the specific subset of conditions of the assay $\mathbf{c}_{\mathbf{j}}$ we want to predict. In Table 5 , we show some selected values of these averages with $>25$ assays reported. It can be noted that the most populated assays in Homo sapiens in the dataset were those in vitro assays that targeted the protein $\mathrm{O} 75874$ (IDH1) and that targeted the cell line U2OS. Upon inspecting Table 5, we can see that $\left\langle D_{k}\left(\mathbf{c}_{\mathbf{j}}\right)\right\rangle$ values change for different subsets of conditions $\mathbf{c}_{j}$. Consequently, when we substitute the different $\left\langle D_{k}\left(\mathbf{c}_{\mathbf{j}}\right)\right\rangle$ values into the model for the same compound, we can calculate different scores $f\left(v_{\mathrm{ij}}\right)_{\text {calc }}$ of biological activity of the same compound under multiple assay conditions. The full list of the values of $\left\langle D_{k}\left(\mathbf{c}_{\mathbf{j}}\right)\right\rangle$ appears in Table S3.

\section{CONCLUSIONS}

In this research work, we generated a PTML-LDA model constructed with antisarcoma assays obtained from ChEMBL and a heterogeneous set of different cell lines, organisms, and targets. As far as we know, this constitutes the first time that this kind of model was tested for sarcoma comprising 34,955 chemical compounds and 37,919 assays. The PTML-LDA model was compared with classic ML approaches like the neural network and also with non-PT consideration. The rate of true positives and true negatives is similar when comparing PTML-LDA to other prediction models. PTML-LDA reduces the amount of input variables (ALOGP and PSA) needed, thus increasing the simplicity and interpretability of the model.

\section{METHODS}

ChEMBL Data Curation and Preprocessing. In total, we downloaded $>370,000$ outcomes for preclinical assays of antisarcoma drug candidates from the ChEMBL database. The keywords (fields) used for the search were as follows: Sarcoma (Assay) and also keywords for more relevant cell osteosarcoma lines MG-63, U2O2, HOS, SAOS-2, and 143B. After that, we carried out a data fusion of the datasets obtained into one single raw dataset. The working dataset was curated by eliminating all duplicated entries. We also eliminated all cases with missing values of biological activity $\left(v_{\mathrm{ij}}\right)$ and/or molecular descriptors. The molecular descriptors used were the same as those precalculated by the ChEMBL database where $D_{1}=\log P$ and $D_{2}=$ PSA. ${ }^{13,14}$ The final dataset obtained after curation contained 37,919 cases comprising 36 protein targets, 43 cell lines, and 17 assay organisms (Table S1). For comparison and exploration with other models, we additionally computed 12 BCUT molecular descriptors ${ }^{64}$ with ChemAxon (http://www.chemaxon.com). The classical unweighted Burden descriptors as well as those weighted by charge and hydrogen bond properties were calculated. The lowest and the three highest eigenvalues were used for descriptor calculation.

To train the model, we split this dataset into two data subsets: training and validation series. We performed a random, stratified, and representative selection of training/ validation cases. To accomplish this task, we sorted the cases by $n_{\mathfrak{j}}$ (from highest to lowest) as well as by assay conditions: biological activity, protein accession, cell line, and assay organism (alphabetically from A to Z). After this, we selected every fourth case ( 1 out of 4 ) to form a training subset $(75 \%$ of cases) and validation subset (25\% of cases). The result of each experimental assay is the value obtained from the quantification of each biological activity and named $v_{\mathrm{ij}}$ (“i” and " $\mathrm{j}$ " represent the assay and conditions, respectively). Each biological activity depends on the conditions $c_{j}\left(c_{0}, c_{1}, c_{2}, \ldots\right.$, $c_{n}$ ) used in each assay. Thus, the conditions taken into account in the data preprocessing were $c_{0}=$ biological activity, $c_{1}=$ protein accession, $c_{2}=$ cell line, and $c_{3}=$ assay organism. From $v_{\mathrm{ij}}$, each experimental assay was discretized based on the desirability $d\left(c_{0}\right)$. This variable was defined as 1 when the result of the desired biological activity depended on an increased value of $v_{\mathrm{ij}}$ and -1 when the desired biological activity depended on a lower value of $v_{\mathrm{ij}}$. Thus, the discretized value $f\left(v_{\mathrm{ij}}\right)_{\text {obs }}$ was calculated as follows: $f\left(v_{\mathrm{ij}}\right)_{\mathrm{obs}}=1$ when $v_{\mathrm{ij}}>$ cut-off and $d\left(\mathrm{c}_{0}\right)=1$. The function $f\left(v_{\mathrm{ij}}\right)_{\mathrm{obs}}=1$ when $v_{\mathrm{ij}}<$ cutoff and $d\left(\mathrm{c}_{0}\right)=-1$; otherwise, $f\left(v_{\mathrm{ij}}\right)_{\mathrm{obs}}=0$. The value $f\left(v_{\mathrm{ij}}\right)_{\mathrm{obs}}=$ 1 refers to a strong effect of the compound over the target. Since $d\left(\mathrm{c}_{0}\right)$ has a direct relationship with $f\left(v_{\mathrm{ij}}\right)_{\text {obs }}$, we applied a rational cut-off for each $c_{0}$, which will be discussed later. Briefly, the cut-off for properties related to drug concentrations and described in $\mathrm{nM}$ (potency, $\mathrm{IC}_{50}, \mathrm{CC}_{50}, \mathrm{EC}_{50}, \mathrm{GI}_{50}$, etc.) was set at 100. For properties described in \% (inhibition, activity, TGI, among others), the cut-off was set at 50. Last, to calculate the probability of these expected values, we evaluated the relationship between the total number of the observed $n\left(f\left(v_{\mathrm{ij}}\right)=1\right)_{\text {obs }}$ within the level of biological activity desired for the condition $c_{j}$ and the total number of compounds $n_{j}$ that were described in that same condition. In this sense, we have that $p\left(f\left(v_{\mathrm{ij}}\right)_{\mathrm{obs}}=1\right)_{\text {expt }}=n\left(f\left(v_{\mathrm{ij}}\right)=1\right)_{\mathrm{obs}} / \mathrm{c}_{0}$.

PTML Linear Model. The multicondition moving averages (MMAs) are PTOs similar to Box-Jenkins moving average operators. However, MMAs are PTOs accounting for perturbations (changes) in multiple conditions $c_{j}$ at the same time, while MA quantifies changes in only one condition. By using linear discriminant analysis (LDA), ${ }^{65}$ we obtained a PTML-LDA equation as follows

$$
\begin{aligned}
& f\left(v_{\mathrm{ij}}\right)_{\mathrm{calc}}= a_{0}+a_{1} \cdot f\left(v_{\mathrm{ij}}\right)_{\mathrm{ref}}+\sum_{k=1}^{k_{\max }} a_{k j} \cdot D_{k}+\sum_{k=1, j=0}^{k_{\max } j_{\max }} a_{k j} \cdot \\
& \Delta D_{k}\left(\mathbf{c}_{\mathrm{j}}\right)
\end{aligned}
$$

The model generates an output score $f\left(v_{\mathrm{ij}}\right)_{\text {calc }}$ that refers to a score function for a biological activity $v_{\mathrm{ij}}$ under the assay conditions $\mathbf{c}_{\mathrm{j}}$. The LDA algorithm includes the Mahalanobis' distance metric, ${ }^{65}$ which makes it possible to infer predictive values through a probability calculation $p\left(f\left(v_{\mathrm{ij}}\right)=1\right)_{\text {pred. }}$. For the variable selection, we detected specific perturbations within the conditions $c_{j}$ that will be adjusted to anticancer properties through a forward-stepwise strategy. ${ }^{65}$ Such conditions as $c_{1}=$ protein accession, $c_{2}=$ cell line, and $c_{3}=$ assay organism were significant, so we took them into consideration in our model. Through $p\left(f\left(v_{\mathrm{ij}}\right)=1\right)_{\text {pred, }}$ we predicted the activity of each compound by applying the function $f\left(v_{\mathrm{ij}}\right)_{\text {pred }}=1$ when $p\left(f\left(v_{\mathrm{ij}}\right)\right.$ $=1)_{\text {pred }}>0.5$ or $f\left(v_{\mathrm{ij}}\right)_{\text {pred }}=0$.

For comparison, we also used a strategy that is not based on perturbation theory. In this sense, besides the molecular descriptors, we added conditions $c_{1}, c_{2}$, and $c_{3}$ as a separate set of categorical variables. A total of 237 variables were needed to represent all conditions. Filtering using the variance of each 
variable leads to a total of 162 variables, including ALOGP and PSA.

The evaluation of the discriminant model was calculated from Wilks' lambda $(\Lambda)$ as follows

$$
\Lambda=\left\lceil\frac{1}{1+\lambda}\right\rceil
$$

where $\Lambda$ is chi-square distributed for $\mathrm{df}=(k-1), k$ is equal to the number of parameters estimated, and $\lambda=\left[\frac{\sum\left(Z_{j}-Z\right)^{2}}{\sum\left(Z_{i j}-Z_{i}\right)^{2}}\right]$.

For ML, besides LDA, we also used neural networks (NN) with different architectures. STATISTICA software was used in both cases. The final networks obtained were multilayer perceptron (MLP), linear neural network (LNN), and radial basis function network (RBF). All these ML strategies were applied with perturbation and nonperturbation theory. The predicted 1 or 0 values were used to determine the specificity or true-negative rate $(S p)$, sensitivity or true-positive rate $(S n)$, and accuracy (Ac) when compared to the observed values. Thus, when $f\left(v_{\mathrm{ij}}\right)_{\text {pre }}=f\left(v_{\mathrm{ij}}\right)_{\text {obs }}$, the cases were determined to be correct. $^{65}$

The metrics to evaluate the performance of all the prediction models were Ac, Sn, and Sp using the following formulae

$$
\begin{aligned}
& \text { Ac }=\frac{\text { number of correctly classified compounds }}{\text { total number of compounds }} \\
& \text { Sn }=\frac{\text { number of correctly classified active compounds }}{\text { total number of active compounds }} \\
& \text { Sp }=\frac{\text { number of correctly classified inactive compounds }}{\text { total number of inactive compounds }}
\end{aligned}
$$

\section{ASSOCIATED CONTENT}

\section{SI Supporting Information}

The Supporting Information is available free of charge at https://pubs.acs.org/doi/10.1021/acsomega.0c03356.

ChEMBL dataset of antisarcoma preclinical experimental assays for the PTML model; results of the analyzed models for sarcoma biological activities; all the multiplecondition averages for all sarcoma assays (XLSX)

\section{AUTHOR INFORMATION}

\section{Corresponding Authors}

Alejandro Cabrera-Andrade - Grupo de BioQuimioinformática and Carrera de Enfermería, Facultad de Ciencias de la Salud, Universidad de Las Américas, Quito 170125, Ecuador; RNASA-IMEDIR, Computer Sciences Faculty, University of A Coruña, A Coruña 15071, Spain; ๑ orcid.org/0000-0001-9702-6618; Email: raul.cabrera@ udla.edu.ec

Humbert González-Díaz - Department of Organic Chemistry II and Basque Center for Biophysics, University of Basque Country UPV/EHU, Leioa 48940, Biscay, Spain; Ikerbasque, Basque Foundation for Science, Bilbao 48011, Biscay, Spain; (1) orcid.org/0000-0002-9392-2797;

Email: humberto.gonzalezdiaz@ehu.es

\section{Authors}

Andrés López-Cortés - RNASA-IMEDIR, Computer Sciences Faculty, University of A Coruña, A Coruña 15071, Spain;
Centro de Investigación Genética y Genómica, Facultad de Ciencias de la Salud Eugenio Espejo, Universidad UTE, Quito 170129, Ecuador

Cristian R. Munteanu - RNASA-IMEDIR, Computer Sciences Faculty, University of A Coruña, A Coruña 15071, Spain; Biomedical Research Institute of A Coruña (INIBIC), University Hospital Complex of A Coruña (CHUAC), A Coruña 15006, Spain; Centro de Investigación en Tecnologias de la Información y las Comunicaciones (CITIC), Campus de Elviña s/n, A Coruña 15071, Spain; ○ orcid.org/0000-00025628-2268

Alejandro Pazos - RNASA-IMEDIR, Computer Sciences Faculty, University of A Coruña, A Coruña 15071, Spain; Biomedical Research Institute of A Coruna (INIBIC), University Hospital Complex of A Coruña (CHUAC), A Coruña 15006, Spain

Yunierkis Pérez-Castillo - Grupo de Bio-Quimioinformática and Escuela de Ciencias Físicas y Matemáticas, Universidad de Las Américas, Quito 170125, Ecuador

Eduardo Tejera - Grupo de Bio-Quimioinformática and Facultad de Ingeniería y Ciencias Aplicadas, Universidad de Las Américas, Quito 170125, Ecuador

Sonia Arrasate - Department of Organic Chemistry II and Basque Center for Biophysics, University of Basque Country UPV/EHU, Leioa 48940, Biscay, Spain

Complete contact information is available at: https://pubs.acs.org/10.1021/acsomega.0c03356

\section{Author Contributions}

${ }^{\&}$ A.C.-A. and A.L.-C. contributed equally to the study.

\section{Notes}

The authors declare no competing financial interest.

\section{ACKNOWLEDGMENTS}

The authors acknowledge research grants from Ministry of Economy and Competitiveness, MINECO, Spain (FEDER CTQ2016-74881-P), and Basque government (IT1045-16). The authors also acknowledge the support of Ikerbasque, Basque Foundation for Science. This work was supported by Universidad de Las Américas and the Collaborative Project in Genomic Data Integration (CICLOGEN) PI17/01826 funded by the Carlos III Health Institute from the Spanish National Plan for Scientific and Technical Research and Innovation 2013-2016 and the European Regional Development Funds (FEDER)-“A way to build Europe". This project was also supported by the General Directorate of Culture, Education and University Management of Xunta de Galicia ED431D 2017/16 and "Drug Discovery Galician Network" ref. ED431G/01 and the "Galician Network for Colorectal Cancer Research” (ref. ED431D 2017/23) and finally by the Spanish Ministry of Economy and Competitiveness for its support through the funding of the unique installation BIOCAI (UNLC08-1E-002, UNLC13-13-3503) and the European Regional Development Funds (FEDER) by the European Union. Additional support was offered by the Consolidation and Structuring of Competitive Research Units-Competitive Reference Groups (ED431C 2018/49), funded by the Ministry of Education, University and Vocational Training of the Xunta de Galicia endowed with EU FEDER funds. 


\section{REFERENCES}

(1) Bray, F.; Ferlay, J.; Soerjomataram, I.; Siegel, R. L.; Torre, L. A.; Jemal, A. Global cancer statistics 2018: GLOBOCAN estimates of incidence and mortality worldwide for 36 cancers in 185 countries. CA Cancer J. Clin. 2018, 68, 394-424.

(2) Hui, J. Y. C. Epidemiology and Etiology of Sarcomas. Surg. Clin. North Am. 2016, 96, 901-914.

(3) Sidaway, P. Sarcoma: Genetic determinants of sarcoma risk revealed. Nat. Rev. Clin. Oncol. 2016, 13, 590.

(4) Thomas, D. M.; Ballinger, M. L. Etiologic, environmental and inherited risk factors in sarcomas. J. Surg. Oncol. 2015, 111, 490-495.

(5) HaDuong, J. H.; Martin, A. A.; Skapek, S. X.; Mascarenhas, L. Sarcomas. Pediatr. Clin. North Am. 2015, 62, 179-200.

(6) Yang, J.; Ren, Z.; Du, X.; Hao, M.; Zhou, W. The role of mesenchymal stem/progenitor cells in sarcoma: update and dispute. Stem Cell Investig. 2014, 1, 18.

(7) Double, J.; Barrass, N.; Barnard, N. D.; Navaratnam, V. Toxicity testing in the development of anticancer drugs. Lancet. Oncol. 2002, 3, 438-442.

(8) Yap, T. A.; Sandhu, S. K.; Workman, P.; de Bono, J. S. Envisioning the future of early anticancer drug development. Nat. Rev. Cancer 2010, 10, 514-523.

(9) Williams, R. J.; Walker, I.; Takle, A. K. Collaborative approaches to anticancer drug discovery and development: a Cancer Research UK perspective. Drug Discovery Today 2012, 17, 185-187.

(10) Heinemann, F.; Huber, T.; Meisel, C.; Bundschus, M.; Leser, $\mathrm{U}$. Reflection of successful anticancer drug development processes in the literature. Drug Discovery Today 2016, 21, 1740-1744.

(11) Sun, J.; Wei, Q.; Zhou, Y.; Wang, J.; Liu, Q.; Xu, H. A systematic analysis of FDA-approved anticancer drugs. BMC Syst. Biol. 2017, 11, 87.

(12) Carvalho-Silva, D.; Pierleoni, A.; Pignatelli, M.; Ong, C.; Fumis, L.; Karamanis, N.; Carmona, M.; Faulconbridge, A.; Hercules, A.; McAuley, E.; Miranda, A.; Peat, G.; Spitzer, M.; Barrett, J.; Hulcoop, D. G.; Papa, E.; Koscielny, G.; Dunham, I. Open Targets Platform: new developments and updates two years on. Nucleic Acids Res. 2019, 47, D1056-D1065.

(13) Mendez, D.; Gaulton, A.; Bento, A. P.; Chambers, J.; De Veij, M.; Félix, E.; Magariños, M. P.; Mosquera, J. F.; Mutowo, P.; Nowotka, M.; Gordillo-Marañón, M.; Hunter, F.; Junco, L.; Mugumbate, G.; Rodriguez-Lopez, M.; Atkinson, F.; Bosc, N.; Radoux, C. J.; Segura-Cabrera, A.; Hersey, A.; Leach, A. R. ChEMBL: towards direct deposition of bioassay data. Nucleic Acids Res. 2019, 47, D930-D940.

(14) Gaulton, A.; Hersey, A.; Nowotka, M.; Bento, A. P.; Chambers, J.; Mendez, D.; Mutowo, P.; Atkinson, F.; Bellis, L. J.; Cibrián-Uhalte, E.; Davies, M.; Dedman, N.; Karlsson, A.; Magariños, M. P.; Overington, J. P.; Papadatos, G.; Smit, I.; Leach, A. R. The ChEMBL database in 2017. Nucleic Acids Res. 2017, 45, D945-D954.

(15) Lo, Y.-C.; Rensi, S. E.; Torng, W.; Altman, R. B. Machine learning in chemoinformatics and drug discovery. Drug Discovery Today 2018, 23, 1538-1546.

(16) Ali, M.; Aittokallio, T. Machine learning and feature selection for drug response prediction in precision oncology applications. Biophys. Rev. 2019, 11, 31-39.

(17) Wang, J.; Yun, D.; Yao, J.; Fu, W.; Huang, F.; Chen, L.; Wei, T.; Yu, C.; Xu, H.; Zhou, X.; Huang, Y.; Wu, J.; Qiu, P.; Li, W. Design, synthesis and QSAR study of novel isatin analogues inspired Michael acceptor as potential anticancer compounds. Eur. J. Med. Chem. 2018, 144, 493-503.

(18) Pogorzelska, A.; Sławiński, J.; Żołnowska, B.; Szafrański, K.; Kawiak, A.; Chojnacki, J.; Ulenberg, S.; Zielińska, J.; Bączek, T. Novel 2-(2-alkylthiobenzenesulfonyl)-3-(phenylprop-2-ynylideneamino)guanidine derivatives as potent anticancer agents - Synthesis, molecular structure, QSAR studies and metabolic stability. Eur. J. Med. Chem. 2017, 138, 357-370.

(19) Sławiński, J.; Szafrański, K.; Pogorzelska, A.; Żołnowska, B.; Kawiak, A.; Macur, K.; Belka, M.; Bączek, T. Novel 2-benzylthio-5(1,3,4-oxadiazol-2-yl)benzenesulfonamides with anticancer activity:
Synthesis, QSAR study, and metabolic stability. Eur. J. Med. Chem. 2017, 132, 236-248.

(20) Singh, H.; Kumar, R.; Singh, S.; Chaudhary, K.; Gautam, A.; Raghava, G. P. S. Prediction of anticancer molecules using hybrid model developed on molecules screened against NCI-60 cancer cell lines. BMC Cancer 2016, 16, 77.

(21) Toropov, A. A.; Toropova, A. P.; Benfenati, E.; Gini, G.; Leszczynska, D.; Leszczynski, J. SMILES-based QSAR approaches for carcinogenicity and anticancer activity: comparison of correlation weights for identical SMILES attributes. Anti-Cancer Agents Med. Chem. 2011, 11, 974-982.

(22) González-Díaz, H.; Bonet, I.; Terán, C.; De Clercq, E.; Bello, R.; García, M. M.; Santana, L.; Uriarte, E. ANN-QSAR model for selection of anticancer leads from structurally heterogeneous series of compounds. Eur. J. Med. Chem. 2007, 42, 580-585.

(23) González-Díaz, H.; Viña, D.; Santana, L.; de Clercq, E.; Uriarte, E. Stochastic entropy QSAR for the in silico discovery of anticancer compounds: prediction, synthesis, and in vitro assay of new purine carbanucleosides. Bioorg. Med. Chem. 2006, 14, 1095-1107.

(24) Gonzáles-Díaz, H.; Gia, O.; Uriarte, E.; Hernádez, I.; Ramos, R.; Chaviano, M.; Seijo, S.; Castillo, J. A.; Morales, L.; Santana, L.; Akpaloo, D.; Molina, E.; Cruz, M.; Torres, L. A.; Cabrera, M. A. Markovian chemicals "in silico" design (MARCH-INSIDE), a promising approach for computer-aided molecular design I: discovery of anticancer compounds. J. Mol. Model. 2003, 9, 395-407.

(25) Jung, M.; Kim, H.; Kim, M. Chemical genomics strategy for the discovery of new anticancer agents. Curr. Med. Chem. 2003, 10, 757762.

(26) Shi, L. M.; Fan, Y.; Myers, T. G.; O’Connor, P. M.; Paull, K. D.; Friend, S. H.; Weinstein, J. N. Mining the NCI anticancer drug discovery databases: genetic function approximation for the QSAR study of anticancer ellipticine analogues. J. Chem. Inf. Comput. Sci. 1998, 38, 189-199.

(27) Barretina, J.; Caponigro, G.; Stransky, N.; Venkatesan, K.; Margolin, A. A.; Kim, S.; Wilson, C. J.; Lehár, J.; Kryukov, G. V.; Sonkin, D.; Reddy, A.; Liu, M.; Murray, L.; Berger, M. F.; Monahan, J. E.; Morais, P.; Meltzer, J.; Korejwa, A.; Jané-Valbuena, J.; Mapa, F. A.; Thibault, J.; Bric-Furlong, E.; Raman, P.; Shipway, A.; Engels, I. H.; Cheng, J.; Yu, G. K.; Yu, J.; Aspesi, P.; de Silva, M.; Jagtap, K.; Jones, M. D.; Wang, L.; Hatton, C.; Palescandolo, E.; Gupta, S.; Mahan, S.; Sougnez, C.; Onofrio, R. C.; Liefeld, T.; MacConaill, L.; Winckler, W.; Reich, M.; Li, N.; Mesirov, J. P.; Gabriel, S. B.; Getz, G.; Ardlie, K.; Chan, V.; Myer, V. E.; Weber, B. L.; Porter, J.; Warmuth, M.; Finan, P.; Harris, J. L.; Meyerson, M.; Golub, T. R.; Morrissey, M. P.; Sellers, W. R.; Schlegel, R.; Garraway, L. A. The Cancer Cell Line Encyclopedia enables predictive modelling of anticancer drug sensitivity. Nature 2012, 483, 603-607.

(28) Toropov, A. A.; Toropova, A. P.; Benfenati, E.; Gini, G.; Leszczynska, D.; Leszczynski, J. CORAL: classification model for predictions of anti-sarcoma activity. Curr. Top. Med. Chem. 2012, 12, 2741-2744.

(29) Vos, H. I.; Coenen, M. J. H.; Guchelaar, H.-J.; Maroeska, D.; te Loo, D. M. The role of pharmacogenetics in the treatment of osteosarcoma. Drug Discovery Today 2016, 21, 1775-1786.

(30) Vamathevan, J.; Clark, D.; Czodrowski, P.; Dunham, I.; Ferran, E.; Lee, G.; Li, B.; Madabhushi, A.; Shah, P.; Spitzer, M.; Zhao, S. Applications of machine learning in drug discovery and development. Nat. Rev. Drug Discovery 2019, 18, 463-477.

(31) Blázquez-Barbadillo, C.; Aranzamendi, E.; Coya, E.; Lete, E.; Sotomayor, N.; González-Díaz, H. Perturbation theory model of reactivity and enantioselectivity of palladium-catalyzed Heck-Heck cascade reactions. RSC Adv. 2016, 6, 38602-38610.

(32) M Casañola-Martin, G.; Le-Thi-Thu, H.; Pérez-Giménez, F.; Marrero-Ponce, Y.; Merino-Sanjuán, M.; Abad, C.; González-Díaz, H. Multi-output Model with Box-Jenkins Operators of Quadratic Indices for Prediction of Malaria and Cancer Inhibitors Targeting UbiquitinProteasome Pathway (UPP) Proteins. Curr. Protein Pept. Sci. 2016, 17, 220-227. 
(33) Romero-Durán, F. J.; Alonso, N.; Yañez, M.; Caamaño, O.; García-Mera, X.; González-Díaz, H. Brain-inspired cheminformatics of drug-target brain interactome, synthesis, and assay of TVP1022 derivatives. Neuropharmacology 2016, 103, 270-278.

(34) Kleandrova, V. V.; Luan, F.; González-Díaz, H.; Ruso, J. M.; Speck-Planche, A.; Cordeiro, M. N. D. S. Computational Tool for Risk Assessment of Nanomaterials: Novel QSTR-Perturbation Model for Simultaneous Prediction of Ecotoxicity and Cytotoxicity of Uncoated and Coated Nanoparticles under Multiple Experimental Conditions. Environ. Sci. Technol. 2014, 48, 14686-14694.

(35) Luan, F.; Kleandrova, V. V.; González-Díaz, H.; Ruso, J. M.; Melo, A.; Speck-Planche, A.; Cordeiro, M. N. D. S. Computer-aided nanotoxicology: assessing cytotoxicity of nanoparticles under diverse experimental conditions by using a novel QSTR-perturbation approach. Nanoscale 2014, 6, 10623-10630.

(36) Alonso, N.; Caamaño, O.; Romero-Duran, F. J.; Luan, F.; Cordeiro, M. N. D. S.; Yañez, M.; González-Díaz, H.; García-Mera, X. Model for High-Throughput Screening of Multitarget Drugs in Chemical Neurosciences: Synthesis, Assay, and Theoretic Study of Rasagiline Carbamates. ACS Chem. Neurosci. 2013, 4, 1393-1403.

(37) Gonzalez-Diaz, H.; Arrasate, S.; Gomez-SanJuan, A.; Sotomayor, N.; Lete, E.; Besada-Porto, L.; Ruso, J. M. General Theory for Multiple Input-Output Perturbations in Complex Molecular Systems. 1. Linear QSPR Electronegativity Models in Physical, Organic, and Medicinal Chemistry. Curr. Top. Med. Chem. 2013, 13, 1713-1741.

(38) Kleandrova, V. V.; Ruso, J. M.; Speck-Planche, A.; Cordeiro, M. N. D. S. Enabling the Discovery and Virtual Screening of Potent and Safe Antimicrobial Peptides. Simultaneous Prediction of Antibacterial Activity and Cytotoxicity. ACS Comb. Sci. 2016, 18, 490-498.

(39) Speck-Planche, A.; Cordeiro, M. N. D. S. Simultaneous virtual prediction of anti-Escherichia coli activities and ADMET profiles: A chemoinformatic complementary approach for high-throughput screening. ACS Comb. Sci. 2014, 16, 78-84.

(40) Speck-Planche, A.; Cordeiro, M. N. D. S. Speeding up Early Drug Discovery in Antiviral Research: A Fragment-Based in Silico Approach for the Design of Virtual Anti-Hepatitis C Leads. ACS Comb. Sci. 2017, 19, 501-512.

(41) Speck-Planche, A.; Cordeiro, M. N. D. S. Computer-aided discovery in antimicrobial research: In silico model for virtual screening of potent and safe anti-pseudomonas agents. Comb. Chem. High Throughput Screening 2015, 18, 305-314.

(42) Speck-Planche, A.; Cordeiro, M. N. D. S. Erratum to: Fragment-based in silico modeling of multi-target inhibitors against breast cancer-related proteins. Mol. Diversity 2017, 21, 525.

(43) Speck-Planche, A.; Cordeiro, M. N. D. S. Fragment-based in silico modeling of multi-target inhibitors against breast cancer-related proteins. Mol. Diversity 2017, 21, 511-523.

(44) Speck-Planche, A.; Kleandrova, V. V.; Luan, F.; Cordeiro, M. N. D. S. Unified multi-target approach for the rational in silico design of anti-bladder cancer agents. Anti-Cancer Agents Med. Chem. 2013, 13, 791-800.

(45) Speck-Planche, A.; Kleandrova, V. V.; Luan, F.; Cordeiro, M. N. D. S. Chemoinformatics in multi-target drug discovery for anti-cancer therapy: in silico design of potent and versatile anti-brain tumor agents. Anti-Cancer Agents Med. Chem. 2012, 12, 678-685.

(46) Speck-Planche, A.; Kleandrova, V. V.; Luan, F.; Cordeiro, M. N. D. S. Rational drug design for anti-cancer chemotherapy: multi-target QSAR models for the in silico discovery of anti-colorectal cancer agents. Bioorg. Med. Chem. 2012, 20, 4848-4855.

(47) Speck-Planche, A.; Kleandrova, V. V.; Luan, F.; Cordeiro, M. N. D. S. Chemoinformatics in anti-cancer chemotherapy: multi-target QSAR model for the in silico discovery of anti-breast cancer agents. Eur. J. Pharm. Sci. 2012, 47, 273-279.

(48) Cordeiro, M. N.; Speck-Planche, A. Computer-aided drug design, synthesis and evaluation of new anti-cancer drugs. Curr. Top. Med. Chem. 2012, 12, 2703-2704.

(49) Speck-Planche, A.; Kleandrova, V. V.; Luan, F.; Cordeiro, M. N. D. S. Multi-target drug discovery in anti-cancer therapy: fragment- based approach toward the design of potent and versatile anti-prostate cancer agents. Bioorg. Med. Chem. 2011, 19, 6239-6244.

(50) Wei, D.-Q.; Selvaraj, G.; Kaushik, A. C. Computational Perspective on the Current State of the Methods and New Challenges in Cancer Drug Discovery. Curr. Pharm. Des. 2018, 24, 3725-3726.

(51) Bediaga, H.; Arrasate, S.; González-Díaz, H. PTML Combinatorial Model of ChEMBL Compounds Assays for Multiple Types of Cancer. ACS Comb. Sci. 2018, 20, 621-632.

(52) Speck-Planche, A.; Kleandrova, V. V.; Luan, F.; Cordeiro, M. N. D. S. Fragment-based QSAR model toward the selection of versatile anti-sarcoma leads. Eur. J. Med. Chem. 2011, 46, 5910-5916.

(53) Chittchang, M.; Gleeson, M. P.; Ploypradith, P.; Ruchirawat, S. Assessing the drug-likeness of lamellarins, a marine-derived natural product class with diverse oncological activities. Eur. J. Med. Chem. 2010, 45, 2165-2172.

(54) Hansch, C.; Verma, R. P. A QSAR study for the cytotoxic activities of taxoids against macrophage (MPhi)-like cells. Eur. J. Med. Chem. 2009, 44, 274-279.

(55) Roy, K.; Pratim Roy, P. Comparative chemometric modeling of cytochrome 3A4 inhibitory activity of structurally diverse compounds using stepwise MLR, FA-MLR, PLS, GFA, G/PLS and ANN techniques. Eur. J. Med. Chem. 2009, 44, 2913-2922.

(56) Sarkar, A.; Anderson, K. C.; Kellogg, G. E. Computational analysis of structure-based interactions and ligand properties can predict efflux effects on antibiotics. Eur. J. Med. Chem. 2012, 52, 98110.

(57) Sliwoski, G.; Kothiwale, S.; Meiler, J.; Lowe, E. W. Computational methods in drug discovery. Pharmacol. Rev. 2014, 66, 334-395.

(58) Leeson, P. Drug discovery: Chemical beauty contest. Nature 2012, 481, 455-456.

(59) Arnott, J. A.; Planey, S. L. The influence of lipophilicity in drug discovery and design. Expert Opin. Drug Discovery 2012, 7, 863-875.

(60) Yuan, H.; Paskov, I.; Paskov, H.; González, A. J.; Leslie, C. S. Multitask learning improves prediction of cancer drug sensitivity. Sci. Rep. 2016, 6, 31619.

(61) Nikolova, O.; Moser, R.; Kemp, C.; Gönen, M.; Margolin, A. A. Modeling gene-wise dependencies improves the identification of drug response biomarkers in cancer studies. Bioinformatics 2017, 33, 13621369.

(62) Waring, M. J. Lipophilicity in drug discovery. Expert Opin. Drug Discovery 2010, 5, 235-248.

(63) Giaginis, C.; Tsopelas, F.; Tsantili-Kakoulidou, A. The Impact of Lipophilicity in Drug Discovery: Rapid Measurements by Means of Reversed-Phase HPLC. Methods Mol. Biol. 1824, 1824, 217-228.

(64) Burden, F. R. Molecular identification number for substructure searches. J. Chem. Inf. Comput. Sci. 1989, 29, 225-227.

(65) Hill, T.; Lewicki, P. STATISTICS Methods and Applications. A Comprehensive Reference for Science, Industry and Data Mining. In Discriminant Function Analysis; 1st ed.; StatSoft, Inc.: 2006; 155-164. 